\title{
Erattum
}

\section{The Evolution of MHC Diversity by Segmental Duplication and Transposition of Retroelements}

\author{
Jerzy K. Kulski, ${ }^{1}$ Silvana Gaudieri, ${ }^{1}$ Matthew Bellgard, ${ }^{1}$ Lois Balmer, ${ }^{1}$ Keith Giles, ${ }^{1}$ Hidetoshi Inoko, ${ }^{2}$ \\ Roger L. Dawkins ${ }^{1}$ \\ ${ }^{1}$ Centre for Molecular Immunology and Instrumentation and the University of Western Australia, Perth, Western Australia \\ ${ }^{2}$ School of Medicine, Tokai University, Isehara, Kanagawa, Japan
}

Re: J Mol Evol (1997) 45(6):599-609. The address of Matthew Bellgard should be Centre for Molecular Immunology and Instrumentation, the University of Western Australia, Perth, and Department of Information Technology, Murdoch University, Murdoch, Western Australia.

In the first paragraph of Materials and Methods (p. 600), “a YAC clone (T109) ...” should read “. . . a YAC clone (Y109) ...'”

On p. 607, first paragraph, lines 16 and 17, “. . . more than 25 mya (Shih et al. 1989)” should read as “... more than 25 mya (Shih et al. 1991).'

On p. 609 of the References, Shih A, Misra R, Rush MG (1989) etc. should be replaced with Shih A, Coutavas EE, Rush MG (1991) Evolutionary implications of primate endogenous retroviruses. Virology 182:495-502. 